Polymer Characterization 



\section{POLYMER CHARACTERIZATION}

by

PROF. EM. DR. SC. NAT. ELISABETH SCHRÖDER Leipzig

and

DOZENT DR. SC. NAT. GERT MÜLLER

DOZENT DR. SC. NAT. KARL-FRIEDRICH ARNDT Merseburg

With 116 Figures and 90 Tables

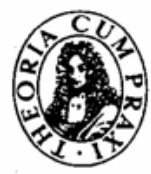

AKADEMIE-VERLAG BERLIN 1989 
The translation was performed by

H. Liebscher $\dagger$ and revised by Dr. John Haim

\section{ISBN 3-05-500538-4}

Erschienen im Akademie-Verlag Berlin, DDR-1086 Berlin, Leipziger Straße 3-4 (C) Akademie-Verlag Berlin 1989

Lizenznummer : $202 \cdot 100 / 475 / 89$

Printed in the German Democratic Republic

Gesamtherstellung: VEB Druckerei „Thomas Müntzer“, 5820 Bad Langensalza LSV 1275

Bestellnummer: 7638562 (6509)

\section{0}

